\title{
Isolated nocturnal desaturation in COPD: prevalence and impact on quality of life and sleep
}

\author{
C A Lewis, ${ }^{1}$ W Fergusson, ${ }^{1}$ T Eaton, ${ }^{1}$ I Zeng, ${ }^{2}$ J Kolbe ${ }^{1,3}$
}

${ }^{1}$ Respiratory Services, Auckland City Hospital, Auckland, New Zealand; ' ${ }^{2}$ Biostatistics Unit, Auckland City Hospital, Auckland, New Zealand; ${ }^{3}$ Department of Medicine, University of Auckland, Auckland, New Zealand

Correspondence to:

Dr C Lewis, Respiratory

Services, Auckland City Hospital,

Grafton, Auckland, New

Zealand; clewis@adhb.govt.nz

Received 15 August 2007 Accepted 16 March 2008 Published Online First 4 April 2008

\section{ABSTRACT}

Background and aims: The clinical impact of nocturnal desaturation on health related quality of life (HROL) and sleep in chronic obstructive pulmonary disease (COPD) has been little studied. The aim of this study was to evaluate the prevalence and clinical impact of nocturnal desaturation in a typical outpatient population with COPD. Patients and methods: Between 2002 and 2005, consecutive patients with COPD attending outpatient services at the study centre underwent resting oximetry if they were not on domiciliary oxygen therapy. If their resting saturations were less than $95 \%$, overnight pulse oximetry was performed. Significant nocturnal desaturation was defined as spending more than $30 \%$ of at least one of two nights with a saturation of less than $90 \%$. The Chronic Respiratory Questionnaire (CRO) and Short Form 36 (SF36) were used to assess HROL, and the Pittsburgh Sleep Quality Index (PSOI), Epworth Sleepiness Score (ESS) and Functional Outcomes of Sleep (FOSO) questionnaires were used to assess sleep quality and daytime function.

Results: Of 1104 patients, 803 underwent resting oximetry and 79 had resting oxygen saturations of less than $95 \%$. Of these, 59 agreed to undergo overnight oximetry (mean age 70 years, forced expiratory volume in $1 \mathrm{~s} 37.2 \%$ predicted, resting $\mathrm{PO}_{2}$ on air $8.9 \mathrm{kPa}$ ). Significant nocturnal desaturation was seen in 29 (49.2\%) of the 59 subjects. Assuming the less hypoxic patients do not have nocturnal desaturation, the prevalence of nocturnal desaturation in the whole clinic population could be estimated at $4.8 \%$. There were no significant differences in CRQ, SF36, PSOI, ESS or FOSO scores for desaturators compared with non-desaturators.

Conclusion: Significant nocturnal desaturation was common in patients with COPD with resting saturations of less than $95 \%$, but was estimated to have a prevalence of less than $5 \%$ in the whole outpatient population. Nocturnal desaturation was not associated with impairment of HROL, sleep quality or daytime function.

Nocturnal desaturation may occur in chronic obstructive pulmonary disease (COPD) in the absence of severe daytime hypoxaemia. The prevalence of such "isolated" nocturnal desaturation has previously been reported at between $25 \%$ and $70 \%{ }^{1-3}$ However, these studies have varied in their inclusion criteria and the definition of nocturnal desaturation used, and therefore uncertainty remains as to the true prevalence of isolated nocturnal desaturation in the stable COPD outpatient population.

Previously, attention focused on the potential adverse effects of nocturnal desaturation on pulmonary haemodynamics in COPD, but a recent study found no difference in pulmonary artery pressures over 2 years in patients with and without nocturnal desaturation. ${ }^{4}$ However, the clinical impact of nocturnal desaturation remains unclear. Four studies have examined the effects of nocturnal desaturation and its correction with oxygen therapy on sleep architecture and quality..$^{5-8}$ These studies were small, short term and sleep laboratory based, employed variable methodology and produced differing results. Only one study attempted to evaluate longer term sleep quality via questionnaires, but aside from this the effects of nocturnal desaturation in COPD on longer term health related quality of life (HROL), sleep quality and daytime function have not been studied. The paucity of data on the prevalence and clinical impact of isolated nocturnal desaturation is reflected in the recent NHLBI research workshop report on supplementary oxygen therapy in COPD, which identified the clinical implications of nocturnal desaturation as one of four areas of oxygen research requiring urgent further study. ${ }^{9}$

The aims of this study were to determine the prevalence and predictors of nocturnal desaturation in a COPD outpatient population, and whether the presence of nocturnal desaturation in patients with COPD was associated with impaired HROL, sleep quality or daytime function. Some of the results from this study have been reported previously in abstract form. ${ }^{10}$

\section{METHODS}

Consecutive patients with COPD attending the outpatient and pulmonary rehabilitation services of Auckland District Health Board (comprising Auckland City Hospital and Green Lane Clinical Centre, Auckland, New Zealand) were screened between December 2002 and December 2005. Eligible subjects had: (1) moderate to severe COPD with forced expiratory volume in $1 \mathrm{~s}$ $\left(\mathrm{FEV}_{1}\right)<60 \%$ predicted and $\mathrm{FEV}_{1} /$ forced vital capacity (FVC) ratio $<70 \%$ predicted; (2) were clinically stable for the previous 4 weeks and receiving usual care; and (3) had a resting saturation of less than $95 \%$ on room air. This last criterion was based: on (1) data from a previous study by our group that showed that patients with saturations of $95 \%$ or greater are very unlikely to have nocturnal desaturation ${ }^{11}$ and (2) the fact that previous studies have shown moderate correlation between daytime oxygenation and nocturnal saturations. ${ }^{2} 1112$

Subjects were excluded if they: (1) were current smokers, (2) were established on domiciliary oxygen therapy, (3) were unable to answer questionnaires and (4) were suspected of having obstructive sleep apnoea syndrome, defined as a 


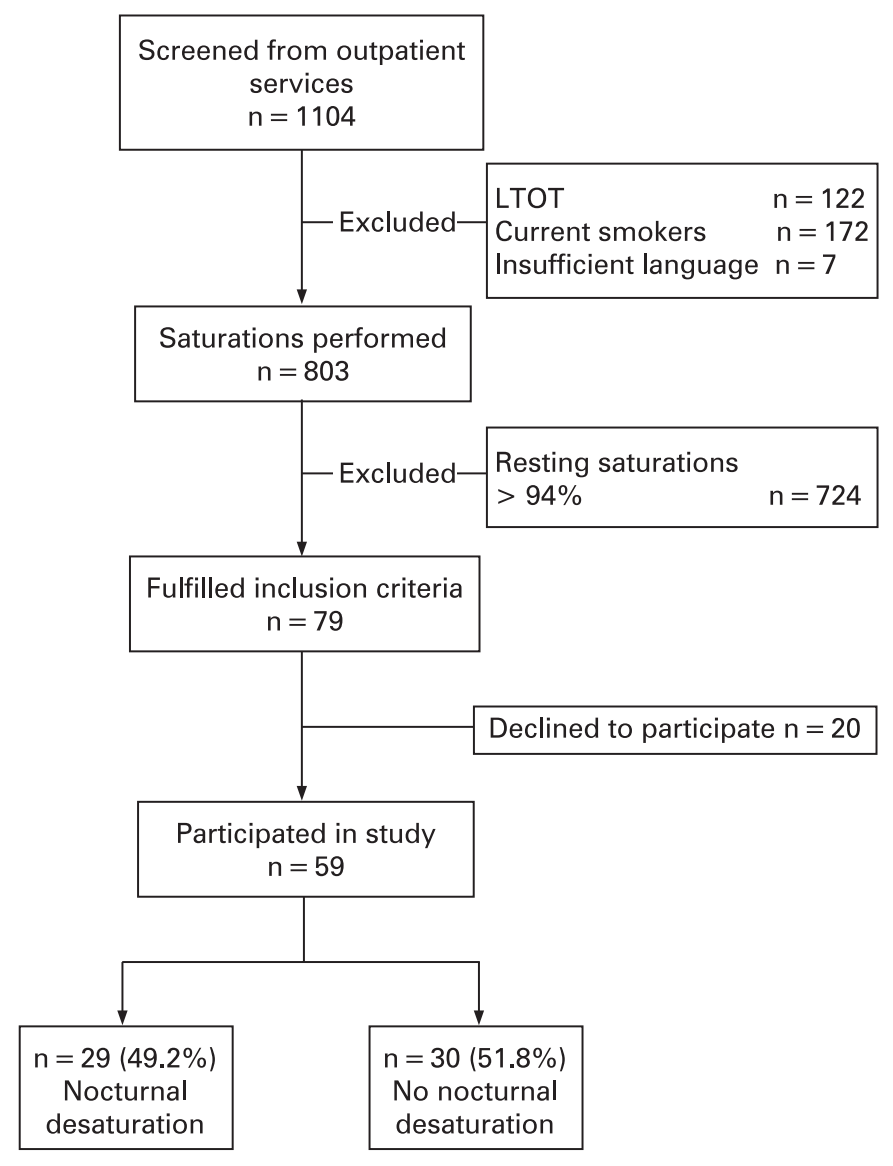

Figure 1 Study design and subjects. LTOT, long term oxygen therapy.

typical clinical history of snoring, witnessed apnoeas, gasping or choking episodes and significant daytime somnolence defined by an Epworth Sleepiness Score of 10 or more. ${ }^{13}$ Written informed consent was obtained from all subjects and the study was approved by the Auckland Ethics Committee.

Subject demographics were collected and body mass index (BMI) was calculated. $\mathrm{FEV}_{1}$ and FVC were measured to ATS standards $^{14}$ using a Microloop portable spirometer (Micro Medical Ltd, Kent, UK) and expressed as per cent predicted using the European Community Coal and Steel prediction equations. ${ }^{15}$ Cutaneous pulse oximetry using a finger probe (Siemens Micro2 plus, Malvern, Pennsylvania, USA) and radial arterial blood gases were taken after the subject had been breathing room air at rest for at least $30 \mathrm{~min}$.

Overnight domiciliary pulse oximetry was carried out on two consecutive nights using a portable oximeter with a finger probe and an $8 \mathrm{~h}$ memory (Siemens Micro2 plus). ${ }^{11}$ Subjects were instructed to commence recording on entering bed to sleep and to discontinue recording once fully awake. Mean nocturnal desaturation (MNS) and the time spend with a saturation below $90 \%$ (TB90\%) were calculated. Subjects with a TB90\% of $30 \%$ or more on at least one of the nights were defined as "desaturators". This definition of significant nocturnal desaturation is accepted internationally, ${ }^{16-18}$ was used in previous studies of nocturnal desaturation ${ }^{34}$ and in our group's study of variability of overnight oximetry. ${ }^{11}$

Each subject completed an interviewer administered Chronic Respiratory Questionnaire (CRO), a well validated measure of disease specific HRQL for which the minimum clinically significant change in score has been established ${ }^{19}{ }^{20}$; the Short
Table 1 Baseline patient characteristics

\begin{tabular}{|c|c|}
\hline & $\begin{array}{l}\text { All patients } \\
(\mathrm{n}=59)\end{array}$ \\
\hline Age (years) & $70(9)$ \\
\hline Male (n (\%)) & $33(56)$ \\
\hline Smoking history (pack-years) & $40.8(26.3)$ \\
\hline $\mathrm{FEV}_{1}$ (I) & $0.9(0.4)$ \\
\hline $\mathrm{FEV}_{1} \%$ predicted & $37.2(14.9)$ \\
\hline FVC (I) & $1.9(0.9)$ \\
\hline FVC\% predicted & $62.1(17.6)$ \\
\hline BMI $\left(\mathrm{kg} / \mathrm{m}^{2}\right)$ & $26.0(6.5)$ \\
\hline Resting $\mathrm{PaO}_{2}(\mathrm{kPa})$ & $8.9(1.2)$ \\
\hline Resting $\mathrm{PacO}_{2}(\mathrm{kPa})$ & $5.6(0.9)$ \\
\hline Resting saturation (\%) & $92.8(1.8)$ \\
\hline Minimum nocturnal saturation (\%) & $86.5(53.6)$ \\
\hline MNS (\%) & $91.2(2.7)$ \\
\hline TB90\% & $34.8(34.9)$ \\
\hline
\end{tabular}

BMl, body mass index; $\mathrm{FEV}_{1}$, forced expiratory volume in $1 \mathrm{~s}$; $\mathrm{FVC}$, forced vital capacity; MNS, mean nocturnal saturation; $\mathrm{PaO}_{2}$, arterial oxygen tension; $\mathrm{PaCO}_{2}$ arterial carbon dioxide tension; TB90\%, time spent with saturation below $90 \%$.

Form 36 (SF36) questionnaire measuring generic $\mathrm{HROL}^{21}$; the Hospital Anxiety and Depression (HAD) questionnaire ${ }^{22}$; the Epworth Sleepiness Score to quantify daytime somnolence ${ }^{13}$; the Functional Outcomes of Sleep (FOSQ) questionnaire, to measure daytime function, outcomes, productivity and vigilance $^{23}$; the Pittsburgh Sleep Quality Index (PSOI), to assess sleep quality and in which "poor" rather than "good" sleep quality is identified by a score greater than $5^{24}$; and Likert ratings of four aspects of their sleep (sleep quality, sleep disturbance, morning refreshment and daytime alertness - see appendix).

\section{Analysis}

Continuous variables are presented as mean (SD) and categorical variables are presented as frequency (percentage) except where stated. Spearman correlation coefficients were used to assess the association between continuous variables. Differences across groups were compared using the Mann-Whitney U test where distribution was skewed. ANCOVA were applied to adjust for covariates in group comparisons and mixed model ANCOVA were used where variance between groups was heterogeneous. Stepwise multiple logistic regressions were used to select the significant predictors for oxygen desaturation. Model assumptions were assessed by residual plots. SAS released 9.1 software (SAS Institute Inc, Cary, North Carolina, USA) was used in the analysis. A p valve $<0.05$ was considered significant.

\section{RESULTS}

\section{Baseline characteristics}

Of 1104 patients attending, 803 suitable patients fulfilled criteria for resting pulse oximetry and of these, 79 had resting saturations of less than $95 \%$ (fig 1). The demographic and clinical details of the 59 subjects who consented to further participation are shown in table 1 . These 59 subjects did not differ significantly in terms of age, $\mathrm{FEV}_{1}$, BMI or resting oxygen saturation from the 20 who declined to participate (data not shown). The group MNS was $91.2 \%$ and TB90\% was $33.2 \%$ during the two nights recorded.

\section{Prevalence of isolated nocturnal desaturation}

Significant nocturnal desaturation (with a TB90\% of greater than $30 \%$ on at least one of two nights) was observed in 29 
Table 2 Baseline patient characteristics: comparison of desaturators and non-desaturators

\begin{tabular}{|c|c|c|c|}
\hline Demographics & $\begin{array}{l}\text { Desaturators } \\
(\mathrm{n}=29)\end{array}$ & $\begin{array}{l}\text { Non-desaturators } \\
(\mathrm{n}=\mathbf{3 0})\end{array}$ & p Value \\
\hline Age (years) & $71(9.1)$ & $70(9.3)$ & 0.71 \\
\hline Male (n (\%)) & $14(48 \%)$ & $19(63 \%)$ & 0.24 \\
\hline Smoking history (pack-years) & $36.4(24.3)$ & $45.0(27.9)$ & 0.17 \\
\hline $\mathrm{FEV}_{1}$ (I) & $0.8(0.5)$ & $0.9(0.4)$ & 0.13 \\
\hline $\mathrm{FEV}_{1}$ (\% predicted) & $35.7(15.1)$ & $38.7(14.8)$ & 0.40 \\
\hline FVC (I) & $1.7(0.8)$ & $2.1(0.8)$ & 0.04 \\
\hline FVC (\% predicted) & $57.8(17.5)$ & $66.2(17.1)$ & 0.04 \\
\hline BMI $\left(\mathrm{kg} / \mathrm{m}^{2}\right)$ & $26.4(5.4)$ & $25.6(7.4)$ & 0.74 \\
\hline Resting $\mathrm{PaO}_{2}(\mathrm{kPa})$ & $8.4(1.2)$ & $9.4(1.1)$ & 0.005 \\
\hline Resting $\mathrm{PacO}_{2}(\mathrm{kPa})$ & $5.9(0.9)$ & $5.2(0.7)$ & 0.005 \\
\hline Resting saturation (\%) & $92.2(2.2)$ & $93.5(0.9)$ & 0.01 \\
\hline MNS (night 1) $(\%)^{*}$ & $90(88,91)$ & $93(92,94)$ & $<0.0001$ \\
\hline MNS (night 2) $(\%)^{*}$ & $89(87,90)$ & $93(92,94)$ & $<0.0001$ \\
\hline TB90\% (night 1)* & $67(36,88)$ & $3.9(0.4,8.2)$ & $<0.0001$ \\
\hline TB90\% (night 2)* & $74(64,94)$ & $2.0(0.2,6.0)$ & $<0.0001$ \\
\hline Minimum nocturnal saturation (night 1$)^{*}(\%)$ & $80(72,83)$ & $86(81,88)$ & $<0.0001$ \\
\hline Minimum nocturnal saturation (night 2$)^{*}(\%)$ & $78(72,83)$ & $84(82,88)$ & 0.0002 \\
\hline
\end{tabular}

Data are presented as mean (SD) unless otherwise stated.

*Median (IOR).

$\mathrm{BMI}$, body mass index; $\mathrm{FEV}_{1}$, forced expiratory volume in $1 \mathrm{~s}$; $\mathrm{FVC}$, forced vital capacity; MNS, mean nocturnal saturation; $\mathrm{PaO}_{2}$, arterial oxygen tension; $\mathrm{PaCO}_{2}$, arterial carbon dioxide tension; TB90\%, time spent with saturation below $90 \%$.

Table 3 Comparison of sleep quality and HROL for desaturators and non-desaturators

\begin{tabular}{|c|c|c|c|c|}
\hline & $\begin{array}{l}\text { All patients } \\
(n=59)\end{array}$ & $\begin{array}{l}\text { Desaturators } \\
(\mathrm{n}=29)\end{array}$ & $\begin{array}{l}\text { Non-desaturators } \\
(\mathrm{n}=\mathbf{3 0})\end{array}$ & p Value* \\
\hline \multicolumn{5}{|l|}{ PSOI } \\
\hline Total score & $7(4,11)$ & $8(4,11)$ & $7(4,11)$ & 0.63 \\
\hline \multicolumn{5}{|l|}{$\mathrm{CRO} \dagger$} \\
\hline Dyspnoea & $16.2(14.8,17.7)$ & $16.3(14.2,18.3)$ & $16.2(14.1,18.3)$ & 0.93 \\
\hline Emotional & $32.9(30.9,34.9)$ & $33.5(30.3,36.6)$ & $32.3(29.7,35.0)$ & 0.41 \\
\hline Fatigue & $15.5(14.3,16.7)$ & $16.0(14.1,18.0)$ & $15.0(13.4,16.5)$ & 0.51 \\
\hline Mastery & $19.4(18.2,20.6)$ & $19.7(17.6,21.7)$ & $19.1(17.7,20.5)$ & 0.41 \\
\hline \multicolumn{5}{|l|}{ SF36 } \\
\hline Physical functioning & $25(10,50)$ & $40(15,55)$ & $17.5(10,40)$ & 0.16 \\
\hline Role physical & $0(0,50)$ & $25(0,50)$ & $0(0,25)$ & 0.07 \\
\hline Pain & $74(51,100)$ & $84(61,100)$ & $74(42,100)$ & 0.27 \\
\hline General health & $37(25,57)$ & $45(30,62)$ & $35(20,52)$ & 0.03 \\
\hline Vitality & $50(40,65)$ & $55(40,70)$ & $50(40,60)$ & 0.33 \\
\hline Social functioning & $75(50,100)$ & $88(62,100)$ & $75(50,87.5)$ & 0.11 \\
\hline Role emotional & $67(33,100)$ & $100(0,100)$ & $67(33,100)$ & $>0.95$ \\
\hline Mental health & $76(64,84)$ & $80(60,84)$ & $74(68,84)$ & 0.75 \\
\hline Mental score total $\dagger$ & $50.3(50.0,55.3)$ & $50.0(47.6,56.4)$ & $50.7(49.8,56.6)$ & 0.92 \\
\hline Physical score total $\dagger$ & $30.3(28.7,33.1)$ & $33.5(31.6,36.8)$ & $27.2(24.5,30.9)$ & 0.007 \\
\hline \multicolumn{5}{|l|}{ FOSO } \\
\hline General productivity & $3.9(3.5,4.0)$ & $3.9(3.8,4.0)$ & $3.7(3.4,4.0)$ & 0.11 \\
\hline Social outcome & $4.0(3.5,4.0)$ & $4.0(4.0,4.0)$ & $4.0(3.0,4.0)$ & 0.26 \\
\hline Activity level & $3.5(3.1,3.8)$ & $3.7(3.2,3.8)$ & $3.5(3.0,3.8)$ & 0.28 \\
\hline Vigilance & $3.7(3.3,4.0)$ & $3.6(3.3,4.0)$ & $3.8(3.4,4.0)$ & 0.79 \\
\hline Epworth Sleepiness Score & $5(2,8)$ & $5(2,8)$ & $5(2,8)$ & 0.88 \\
\hline \multicolumn{5}{|l|}{ Likert scores } \\
\hline Patients' described sleep quality & $5(4,6)$ & $5(4,5)$ & $5(4,6)$ & 0.69 \\
\hline How disturbed sleep is & $3(2,5)$ & $3(2,5)$ & $3(2,4)$ & 0.47 \\
\hline How refreshed in the morning & $4(2,6)$ & $4(3,6)$ & $4(2,5)$ & 0.63 \\
\hline How alert in day time & $5(4,6)$ & $5(4,5)$ & $5(4,6)$ & 0.87 \\
\hline \multicolumn{5}{|l|}{ HAD } \\
\hline Anxiety score & $6(2,8)$ & $6(2,8)$ & $5.5(3,8)$ & 0.96 \\
\hline Depression score & $4(2,6)$ & $4(2,6)$ & $4(2,7)$ & 0.36 \\
\hline
\end{tabular}

*Mann-Whitney U test. ANCOVA were used to adjust the group comparisons of PSOl, CRO, SF36 summary score total, Epworth Sleepiness Score, sleep quality and HAD by age, gender and FEV $1 \%$ predicted). The results made no difference.

$\dagger$ Data presented are mean $(95 \% \mathrm{Cl})$; the rest of the data are presented as median (IQR).

CRO, Chronic Respiratory Questionnaire; FOSQ, Functional Outcomes of Sleep; HAD, Hospital Anxiety and Depression; HRQL, health related quality of life; PSQI, Pittsburgh Sleep Quality Index; SF36, Short Form 36. 
Table 4 Correlations between nocturnal saturations and daytime parameters

\begin{tabular}{lrr}
\hline Measure & \multicolumn{1}{l}{ MNS } & \multicolumn{1}{l}{ TB90\% } \\
\hline $\mathrm{PaO}_{2}$ & $0.51(p=0.0001)$ & $-0.44(p=0.001)$ \\
$\mathrm{PaCO}_{2}$ & $-0.34(p=0.02)$ & $0.31(p=0.03)$ \\
$\mathrm{FEV}_{1}$ & $0.23(p=0.09)$ & $-0.23(p=0.08)$ \\
$\mathrm{FEV}_{1} \%$ predicted & $0.17(p=0.19)$ & $-0.18(\mathrm{p}=0.18)$ \\
$\mathrm{FVC}$ & $0.23(\mathrm{p}=0.09)$ & $-0.24(\mathrm{p}=0.06)$ \\
$\mathrm{FVC} \%$ predicted & $0.24(\mathrm{p}=0.07)$ & $-0.26(\mathrm{p}=0.05)$ \\
$\mathrm{BMI}$ & $-0.15(\mathrm{p}=0.3)$ & $0.11(\mathrm{p}=0.40)$ \\
Pack-year smoking history & $0.21(\mathrm{p}=0.12)$ & $-0.23(\mathrm{p}=0.08)$ \\
\hline
\end{tabular}

Results are presented as correlation coefficient ( $\mathrm{p}$ value).

$\mathrm{BMI}$, body mass index; $\mathrm{FEV}_{1}$, forced expiratory volume in $1 \mathrm{~s}$; FVC, forced vital capacity; MNS, mean nocturnal saturation; $\mathrm{PaO}_{2}$, arterial oxygen tension; $\mathrm{PaCO}_{2}$, arterial carbon dioxide tension; TB90\%, time spent with saturation below $90 \%$.

(49.2\%) of the 59 subjects, thus giving a prevalence of nocturnal desaturation of $49.2 \%$ in patients with COPD with resting saturations of less than $95 \%$. Assuming, as discussed earlier, nocturnal desaturation was not present in the other patients with saturations of $95 \%$ or greater, the overall prevalence of nocturnal desaturation in the whole non-smoking COPD clinic population could be estimated as $4.8 \%$ (assuming the same prevalence of desaturation in the 20 similar eligible subjects who declined to participate).

\section{Clinical effects of isolated nocturnal desaturation}

Comparing desaturators and non-desaturators (as previously defined), resting oxygen saturation and $\mathrm{PaO}_{2}$ were significantly lower and $\mathrm{PaCO}_{2}$ higher in desaturators. FVC was slightly lower in desaturators but there were no other significant differences between the groups including $\mathrm{FEV}_{1}$, BMI and smoking history (table 2).

Overall sleep quality was poor and there were no significant differences between groups in overall sleep quality, as measured by the PSOI, or in daytime productivity, outcomes, activity or vigilance, as measured by the FOSO, or in Likert scores for any of the four ratings of sleep quality and daytime function (table 3). The Epworth score was not elevated in either group, and there was no significant difference in Epworth score between the groups.

There were no significant differences between groups in disease specific HRQL, as measured by the domains of the CRO, or in Hospital Anxiety and Depression scores (table 3). For the SF36, the physical score and general health scores were significantly better in desaturators but there were no other significant differences between the groups.

\section{Predictors of isolated nocturnal desaturation}

TB90\% and MNS were each significantly correlated with daytime $\mathrm{PO}_{2}$ and $\mathrm{PCO}_{2}$ (table 4). A weak correlation was found between TB90\% and FVC per cent predicted. There was no significant correlation between either $\mathrm{TB} 90 \%$ or $\mathrm{MNS}$ and $\mathrm{FEV}_{1}$, FVC (1), smoking history and BMI.

\section{Variability of nocturnal desaturation}

There was little variation in the overall group MNS, TB90\% and minimum nocturnal desaturation between nights (table 2). However, individual subjects exhibited substantial variation in the degree of desaturation between nights when expressed as TB90\% (fig 2). Eight of the 29 desaturating subjects (27.6\%) exhibited significant nocturnal desaturation on only one of the two nights. These results are very similar to those from a

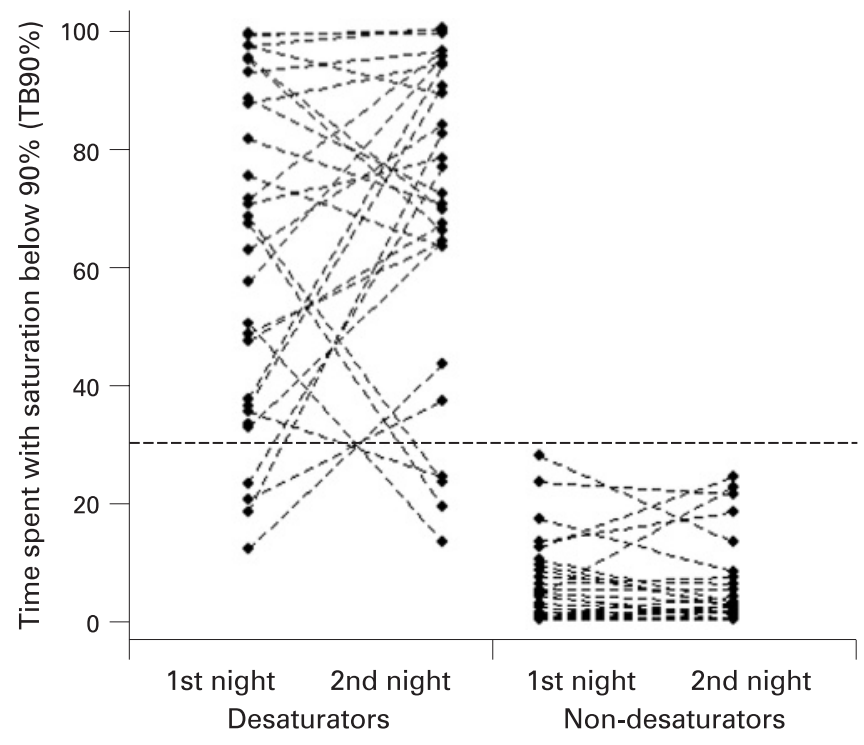

Figure 2 Comparison between desaturators and non-desaturators of time spent with saturation below $90 \%$ (TB90\%) for the first and second nights recorded.

separate study previously reported by our group. ${ }^{11}$ There was also some individual subject variability between minimum saturations recorded on each night (fig 3).

\section{DISCUSSION}

This study demonstrated that the prevalence of isolated nocturnal desaturation in a typical COPD outpatient clinic population is estimated to be very low, at less than $5 \%$. However, just under half of patients with COPD with impaired resting daytime oxygen saturations on air of less than $95 \%$ exhibit isolated nocturnal desaturation, similar to previous studies. We have confirmed moderate correlations between nocturnal desaturation and daytime $\mathrm{PaO}_{2}$ and $\mathrm{PaCO}_{2}$ but not other physiological variables, and that there is variability in the

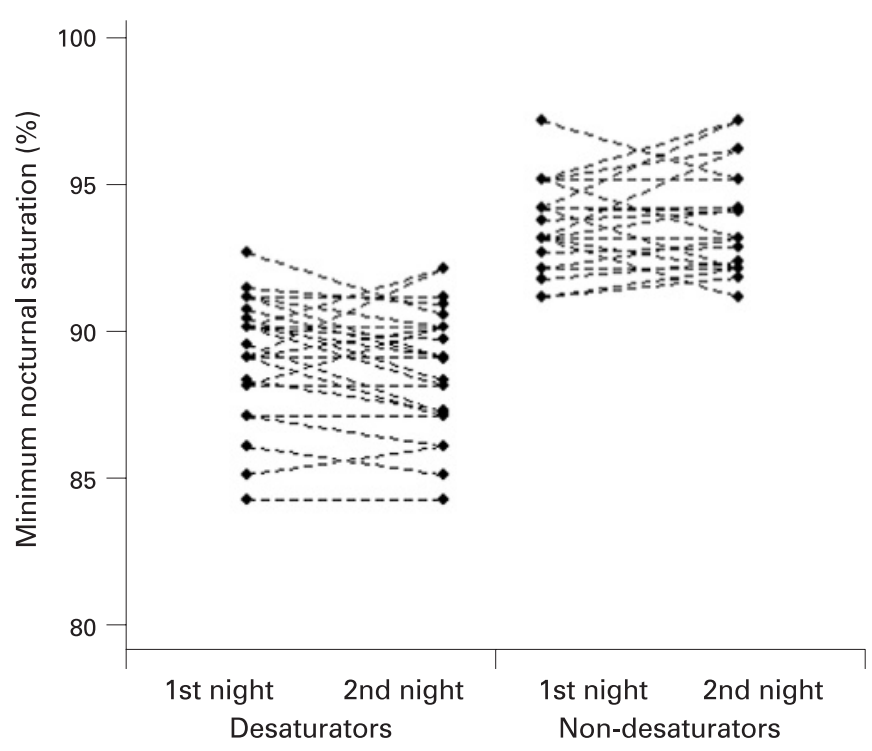

Figure 3 Comparison between desaturators and non-desaturators of minimum recorded nocturnal saturation for the first and second nights recorded. 
degree of nocturnal desaturation exhibited by subjects between nights. We found that isolated nocturnal desaturation, as currently defined, was not associated with impairment of sleep quality, daytime function or health related quality of life.

The prevalence of significant nocturnal desaturation in our study subjects, who had resting saturations of less than $95 \%$ on air and subsequently underwent pulse oximetry, was compatible with previous studies. Fletcher et al studied 135 patients with a $\mathrm{PaO}_{2}$ of greater than $60 \mathrm{~mm} \mathrm{Hg}$ and found nocturnal desaturation in $35(27 \%)$, although nocturnal desaturation was defined differently (a fall below $90 \%$ for 5 min or more, with a nadir saturation below $85 \%$ ). ${ }^{1}$ Vos et al studied 39 patients with a $\mathrm{PaO}_{2}$ of more than $8 \mathrm{kPa}$ and found 10 (25.6\%) were desaturators, defined as a mean nocturnal saturation below $90 \% .^{2}$ In neither of these studies are the subjects stated to be identified through consecutive unselected screening. Most recently, Chaouat et al studied 94 patients with mild to moderate hypoxaemia $\left(\mathrm{PaO}_{2} 7.4-9.2 \mathrm{kPa}\right)$ and found nocturnal desaturation in $66(70.2 \%)$ using the same definition as in this study. ${ }^{3}$

A strength of this study is that, unlike the previous studies outlined above, we consecutively screened an entire COPD outpatient clinic population with resting oximetry. We excluded smokers from this study because in our centre and elsewhere smokers would not be eligible for potential therapeutic intervention with domiciliary oxygen. ${ }^{25}$ We were therefore able to estimate that the overall prevalence of nocturnal desaturation in non-smoking COPD patients not on long term oxygen therapy was less than $5 \%$. This indicates that nocturnal desaturation is a less common clinical problem in practice than would have been suggested by previous studies. We have, of course, had to make two assumptions to produce this estimate. The first is that the prevalence of nocturnal desaturation in the 20 patients who declined oximetry was the same as in the 59 subjects who did have oximetry. As there were no significant differences between these subjects in terms of baseline measures, this assumption seems very reasonable. The second more important assumption we have made is that none of our patients with resting saturations of $95 \%$ or greater who did not undergo oximetry would have had nocturnal desaturation. Given the relationship between $\mathrm{PO}_{2}$ and nocturnal desaturation in both this and previous studies, we believe that the number of patients with significant nocturnal desaturation in the remainder of our clinic population is likely to be very small, and is unlikely to make a meaningful difference to our estimated prevalence of nocturnal desaturation of $4.8 \%$. Furthermore, if nocturnal desaturation were found in patients with higher daytime saturations, it is more likely to be caused by other conditions such as obstructive sleep apnoea.

To our knowledge, this is the first study to examine the relationship between nocturnal desaturation and longer term sleep quality and HROL in a representative COPD population. Previous studies have examined sleep quality of COPD patients in a sleep laboratory setting, compared with normal aged matched controls. Patients with COPD had reduced sleep time, increased sleep stage changes, increased arousal frequency, lower baseline oxygen saturations and episodes of desaturation, often associated with REM sleep. ${ }^{5}$ The correction of nocturnal desaturation with oxygen in these studies did not consistently lead to improvements in sleep architecture or quality-two studies showed improvements in sleep times and decreased arousals, ${ }^{5}{ }^{6}$ but two showed no improvement. ${ }^{78}$ This suggests that impairment of sleep quality in COPD is not solely a result of hypoxaemia or nocturnal desaturation, and is consistent with the lack of difference in sleep quality and HROL between desaturators and non-desaturators in this study. The sleep quality of our COPD subjects irrespective of desaturation was poor as rated by the PSOI score, with a mean total score of 7.3, and 36 of 59 subjects were classified as "poor" sleepers (61\%). A previous study of 44 healthy elderly controls (over 80 years of age) revealed a mean PSOI score of 4.4 in men and 5.1 in women, with only $31.9 \%$ classified as "poor" sleepers. ${ }^{26}$ In the same study, only 1 of 35 young healthy controls (aged 2030 years) was classified as a poor sleeper. Therefore, patients with COPD suffer impaired sleep quality irrespective of nocturnal desaturation, which cannot be accounted for by advanced age alone. Other factors such as cough, bronchospasm, mood disturbance and side effects of medication are likely to adversely impact on sleep quality. It is possible that the "noise" created by these problems also dilutes any "signal" of impaired sleep quality from episodes of desaturation. Finally, as previously, ${ }^{11}$ we have demonstrated that there is considerable variability in the degree of nocturnal desaturation (as TB90\%) between nights in individual subjects. This may in part reflect the nature of the oxyhaemoglobin dissociation curve, in that a small fall in the resting daytime $\mathrm{PO}_{2}$ may lead to more marked desaturation at night. This innate variation may render more difficult the detection of consistent daytime consequences of nocturnal desaturation.

Our study had limitations which warrant discussion. Firstly, it is possible that because of our sample size, a deleterious effect of nocturnal desaturation has been missed. As this was a pragmatic prospective survey of our clinic population, a formal power calculation was not performed. However, we believe that the number of study subjects should have been sufficient to show any meaningful clinical differences in sleep quality or HROL, and our data do not reveal any trends or minor differences in favour of our hypothesis which could have been accentuated by larger numbers. While the HROL questionnaires used are well validated and sensitive, with extensive previous use in COPD research, the sleep questionnaires used are not validated in this setting, and in particular the PSOI was designed as a screening tool for sleep disorders rather than as an outcome measure for clinical trials. It is possible that the PSOI was not sensitive enough to detect differences in sleep quality in this study. However, the PSOI scores showed that the overall sleep quality of our patients was poor, irrespective of nocturnal saturations. Also, the FOSQ has not been validated as a measure of daytime somnolence in this population, and it is notable that the overall Epworth score in our population was not elevated despite the impaired sleep quality, an observation which has been made previously. ${ }^{27}$ It would be appropriate for future COPD therapeutic intervention studies to include measures of sleep quality and the daytime consequences of such, and there is therefore a need for development of a COPD specific sleep questionnaire for use in such studies which is valid, reproducible and responsive to change. Finally, this was not an intervention study. It is possible that even though differences are not apparent at baseline, correction of nocturnal desaturation with oxygen therapy may improve sleep quality and HROL. This would require evaluation in a randomised controlled trial.

In conclusion, in a survey of a typical COPD outpatient population, isolated nocturnal desaturation was very uncommon, but was present in approximately half of patients with resting saturations less than 95\%. Patients with nocturnal desaturation did not appear to have impaired sleep quality, HROL or excess daytime somnolence compared with those 
without desaturation. Our findings suggest that nocturnal desaturation in COPD is an uncommon phenomenon of limited clinical significance.

Acknowledgements: The authors wish to thank Teena West, biostatistician, for her help with the design of this study.

Funding: This study was funded by a grant from the Auckland Medical Research Foundation.

Competing interests: None.

Ethics approval: The study was approved by the Auckland Ethics Committee.

\section{REFERENCES}

1. Fletcher EC, Miller J, Divine GW, et al. Nocturnal oxyhaemoglobin desaturation in COPD patients with arterial oxygen tensions above $60 \mathrm{mmHg}$. Chest 1992:4:604-8.

2. Vos PJE, Folgering HThM, van Herwaarden CLA. Predictors for nocturnal hypoxaemia (mean $\mathrm{SaO2}<90 \%$ ) in normoxic and mildly hypoxic patients with COPD. Eur Respir J 1995;8:74-7.

3. Chaouat A, Weitzenblum E, Kessler R, et al. Sleep-related 02 desaturation and daytime pulmonary haemodynamics in COPD patients with mild hypoxaemia. Eur Respir J 1997:10:1730-5.

4. Chaouat A, Weitzenblum E, Kessler R, et al. Outcome of COPD patients with mild daytime hypoxaemia with or without sleep-related oxygen desaturation. Eur Respir J 2001;17:848-55

5. Calverley PMA, Brezinova V, Douglas NJ, et al. The effect of oxygenation on sleep quality in chronic bronchitis and emphysema. Am Rev Respir Dis 1982;126:206-10.

6. Fleetham J, West P, Mezon B, et al. Sleep, arousals, and oxygen desaturation in chronic obstructive pulmonary disease. The effect of oxygen therapy. Am Rev Respir Dis 1982:126:429-33

7. Goldstein RS, Ramcharan V, Bowes G, et al. Effect of supplemental nocturnal oxygen on gas exchange in patients with severe obstructive lung disease. N Engl J Med 1984:310:425-9.

8. McKeon JL, Murree-Allen K, Saunders NA. Supplemental oxygen and quality of sleep in patients with chronic obstructive lung disease. Thorax 1989:44:184-8.

9. Croxton TL, Bailey WC. Long term oxygen treatment in chronic obstructive pulmonary disease: recommendations for future research. An NHLBI Workshop report. Am J Respir Crit Care Med 2006;174:373-8.

10. Lewis CA, Eaton T, Fergusson W, et al. The prevalence of nocturnal desaturation in COPD patients without daytime hypoxia. Am J Repir Care Med 2007;175:A615.

11. Lewis CA, Eaton T, Fergusson W, et al. Home overnight pulse oximetry in patients with COPD: more than one recording may be needed. Chest 2003;123:1127-33.

12. Connaughton JJ, Catterall JR, Elton RA, et al. Do sleep studies contribute to the management of patients with severe chronic obstructive pulmonary disease? Am Rev Respir Dis 1988;138:341-4.

13. Johns MW. A new method for measuring daytime sleepiness: the Epworth sleepiness scale. Sleep 1991:14:540-5.

14. Standardisation of spirometry: a statement of the American Thoracic Society. Am J Respir Crit Care Med 1996;152:1107-36.

15. Quonier PH. Standardisation of lung function testing. Report of working party. European Community for Coal and Steel recommendations. Bull Eru Physiopathol Respir 1983;19(Suppl 5):1-93.
16. Royal College of Physicians. Domiciliary oxygen therapy services: clinical guidelines and advice for prescribers. A report of the Royal College of Physicians. London: Royal College of Physicians, 1999.

17. Chronic obstructive pulmonary disease. National clinical guideline on management of chronic obstructive pulmonary disease in adults in primary and secondary care. Managing stable COPD. Thorax 2004;59(Suppl 1):i39-130.

18. American Thoracic Society and European Respiratory Society. Standards for the diagnosis and management of patients with COPD. http://dev.ersnet.org/275guidelines.htm (accessed 28 November 2008).

19. Guyatt GH, Berman LB, Townsend $M$, et al. A measure of quality of life for clinical trials in chronic lung disease. Thorax 1987:42:773-8.

20. Jaeschke R, Singer J, Guyatt GH. Measurement of health status: ascertaining the minimal clinically important difference. Control Clin Trials 1989;10:407-15.

21. Brazier JE, Harper R, Jones NMB, et al. Validating the SF-36 health survey questionnaire: new outcome measure for primary care. BMJ 1992;305:160-4

22. Zigmond AS, Snaith RP. The Hospital Anxiety and Depression Scale. Acta Psychiatr Scand 1983;67:361-70.

23. Weaver TE, Laizner AM, Evans LK. An instrument to measure functional status outcomes for disorders of excessive sleepiness. Sleep 1997;20:835-43.

24. Buysse DJ, Reynolds CF, Monk TH, et al. The Pittsburgh Sleep Quality Index: A new instrument for psychiatric practice and research. Psychiatry Res 1989;28:193-213

25. McDonald CF, Crockett AJ, Young $\mathbf{H}$. Adult domiciliary oxygen therapy. Position statement of the Thoracic Society of Australia and New Zealand. Med J Aust 2005; 182:621-6.

26. Buysse DJ, Reynolds CF, Monk TH, et al. Quantification of subjective sleep quality in healthy elderly men and women using the Pittsburgh Sleep Quality Index (PSOI). Sleep 1991:14:331-8.

27. McNicholas WT, Calverley PMA, Lee A, et al. Long acting inhaled anticholinergic therapy improves sleeping oxygen saturation in COPD. Eur Respir $J$ 2004:23:825-31.

\section{APPENDIX}

Likert scores used to measure sleep quality and daytime function are shown in table A1.

Table A1 Likert scores used to measure sleep quality and daytime function

How would you describe your overall sleep quality over the last 4 weeks? $\begin{array}{lllllllll}\text { Extremely poor } & 1 & 2 & 3 & 4 & 5 & 6 & 7 & \text { Extremely good }\end{array}$ How disturbed or interrupted has your sleep been over the last four weeks? $\begin{array}{lllllllll}\text { Not at all } & 1 & 2 & 3 & 4 & 5 & 6 & 7 & \text { Extremely }\end{array}$ How refreshed have you felt in the morning after waking up in the last 4 weeks? $\begin{array}{lllllllll}\text { Not at all } & 1 & 2 & 3 & 4 & 5 & 6 & 7 & \text { Extremely }\end{array}$ How alert have you felt during the daytime over the last four weeks?

$\begin{array}{lllllllll}\text { Not at all } & 1 & 2 & 3 & 4 & 5 & 6 & 7 & \text { Extremely }\end{array}$

\section{Access all our original articles online even before they appear in a print issue!}

Online First is an exciting innovation that allows the latest clinical research papers to go from acceptance to your browser within days, keeping you at the cutting edge of medicine.

Simply follow the Online First link on the homepage and read the latest Online First articles that are available as unedited manuscripts in downloadable PDF form. The articles are peer reviewed, accepted for publication and indexed by PubMed but not yet included in a journal issue, so you'll be among the first to read them! 